Supporting Information

\title{
Acoustic Cavitation Assisted formulation of Solid Lipid Nanoparticles using Different Stabilizers
}

Raj Kumar, ${ }^{*, a, b, \dagger}$, Ashutosh Singh ${ }^{\mathrm{a}, \mathrm{b}, \mathrm{c}}$ and Neha Garg*,a,b,c

a School of Basic Sciences, Indian Institute of Technology Mandi, Mandi-175005, Himachal Pradesh, India.

b Advanced Material Research Centre, Indian Institute of Technology Mandi, Mandi-175005, Himachal Pradesh, India.

${ }^{c}$ Bio-X Research Centre, Indian Institute of Technology Mandi, Mandi-175005, Himachal Pradesh, India

*Corresponding authors:

Dr. Raj Kumar

Department of Pharmaceutical Sciences, University of Michigan, 2800 Plymouth Road, Ann Arbor, 48109-2800, Michigan, United States

Email: rk7410@gmail.com;

Dr. Neha Garg

School of Basic Sciences, Indian Institute of Technology Mandi, Mandi-175005, Himachal Pradesh, India

Email: neha@iitmandi.ac.in;

$\dagger$ Present Address: Department of Pharmaceutical Sciences, University of Michigan, 2800 Plymouth Road, Ann Arbor, 48109-2800, Michigan, United States 
Table S1 Comparison of encapsulation efficiency (\%EE) and drug loading (\%DL) percentage of solid lipid nanoparticles containing Compritol ${ }^{\circledR} 888$ ATO (CPT) and/or ketoprofen (KP) from reported literature.

\begin{tabular}{|c|c|c|c|c|c|c|c|}
\hline Entry & Lipid & Drug & Stabilizer & Process/method & $\begin{array}{l}\text { Particle size } \\
(\mathrm{d} / \mathrm{nm})\end{array}$ & \%EE & $\% \mathrm{ODL}$ \\
\hline 1 & Compritol & Ibuprofen & $\begin{array}{l}\text { Poloxamer }{ }^{\circledR} 188 \\
\text { Tween }{ }^{\circledR} 80\end{array}$ & Hot melt oil in water emulsion ${ }^{1}$ & $\begin{array}{l}87 \mathrm{~nm} \\
70 \mathrm{~nm}\end{array}$ & $\begin{array}{l}-- \\
--\end{array}$ & $\begin{array}{l}-- \\
--\end{array}$ \\
\hline 2 & Compriol & Indomethacin & $\begin{array}{l}\text { Sucrose fatty acid ester } \\
\text { SP } 30\end{array}$ & Hot Homogenization $^{2}$ & $211 \mathrm{~nm}$ & -- & -- \\
\hline \multirow[t]{3}{*}{3} & \multirow[t]{3}{*}{ Compritol } & Stavudine, & $\begin{array}{l}\text { L- } \alpha- \\
\text { phospatidylcholine, }\end{array}$ & Microemulsion $^{3}$ & $258 \mathrm{~nm}$ & $\sim 45$ & -- \\
\hline & & Delavirdine, & $\begin{array}{l}\text { Cholesteryl } \\
\text { hemisuccinate, }\end{array}$ & & $142 \mathrm{~nm}$ & $\sim 65$ & -- \\
\hline & & Saquinavir, & Taurocholate & & $198 \mathrm{~nm}$ & $\sim 74$ & -- \\
\hline 4 & Compritol & $\begin{array}{l}\text { Rosuvastatin } \\
\text { calcium }\end{array}$ & $\begin{array}{l}\text { Tween }{ }^{\circledR} 20 \\
\text { Poloxamer }{ }^{\circledR} 188\end{array}$ & $\begin{array}{l}\text { Hot homogenization followed by } \\
\text { an ultrasonication }{ }^{4}\end{array}$ & $\begin{array}{l}899 \mathrm{~nm} \\
987 \mathrm{~nm}\end{array}$ & $\begin{array}{l}46 \\
51\end{array}$ & $\begin{array}{l}-- \\
--\end{array}$ \\
\hline 5 & Compritol & $\begin{array}{l}\text { Diclofenac } \\
\text { diethylamine }\end{array}$ & $\begin{array}{l}\text { Soya lecithin, } \\
\text { Pluronic } ₫ \text { F68 }\end{array}$ & Solvent emulsion evaporation ${ }^{5}$ & $178 \mathrm{~nm}$ & 54 & -- \\
\hline 6 & Compritol & Lornoxicam & $\begin{array}{l}\text { Lecithin, Sodium } \\
\text { cholate and } \\
\text { Poloxamer }{ }^{\circledR} 188\end{array}$ & $\begin{array}{l}\text { Emulsification solvent } \\
\text { evaporation }{ }^{6}\end{array}$ & $289 \mathrm{~nm}$ & 67 & -- \\
\hline 7 & $\begin{array}{l}\text { Glyceryl } \\
\text { monostearate }\end{array}$ & Ketoprofen & Tween ${ }^{\circledR} 80$ & Emulsion solvent evaporation ${ }^{7}$ & $100 \mu \mathrm{m}$ & 72 & 18 \\
\hline 8 & Capmul & Ketoprofen & Gelucire ${ }^{\circledR}$ 50/13 & $\begin{array}{l}\text { Hot melt homogenization } \\
\text { followed by sonication }{ }^{8}\end{array}$ & $244 \mathrm{~nm}$ & 72 & 02 \\
\hline 9 & Compritol & $\begin{array}{l}\text { Superoxide } \\
\text { dismutase }\end{array}$ & $\begin{array}{l}\text { Tween }{ }^{\circledR} 80 \text { and } \operatorname{Span} \AA \\
20\end{array}$ & Cold homogenization ${ }^{9}$ & $102 \mathrm{~nm}$ & 78 & -- \\
\hline 10 & Compritol & Meloxicam & Poloxamer ${ }^{\circledR} 188$ & $\begin{array}{l}\text { Modified high shear } \\
\text { homogenization and } \\
\text { ultrasonication }{ }^{10}\end{array}$ & $325 \mathrm{~nm}$ & 85 & -- \\
\hline
\end{tabular}




\begin{tabular}{|c|c|c|c|c|c|c|c|}
\hline 11 & Compritol & Carvedilol & Poloxamer ${ }^{\circledR} 188$ & $\begin{array}{l}\text { Emulsion/solvent evaporation } \\
\text { method }{ }^{11}\end{array}$ & $134 \mathrm{~nm}$ & 87 & -- \\
\hline 12 & Compritol & $\begin{array}{l}\text { Diclofenac } \\
\text { sodium }\end{array}$ & $\begin{array}{l}\text { Hydroxypropyl- } \beta- \\
\text { cyclodextrin }\end{array}$ & Oil/water hot homogenization ${ }^{12}$ & $300 \mathrm{~nm}$ & 88 & -- \\
\hline 13 & Compritol & $\begin{array}{l}\text { Montelukast } \\
\text { sodium }\end{array}$ & Polyvinyl alcohol & $\begin{array}{l}\text { Hot homogenization followed by } \\
\text { an ultrasonication }{ }^{13}\end{array}$ & $2.95 \mu \mathrm{m}$ & 92 & -- \\
\hline 14 & Compritol & Lornoxicam & Pluronic $\AA$ F68 & High pressure homogenization ${ }^{14}$ & $185 \mathrm{~nm}$ & 93 & 11 \\
\hline 15 & $\begin{array}{l}\text { Beeswax and } \\
\text { Carnauba } \\
\text { wax }\end{array}$ & Ketoprofen & $\begin{array}{l}\text { Tween }{ }^{\circledR} 20 \text { and egg } \\
\text { lecithin }\end{array}$ & Microemulsion ${ }^{15}$ & $75 \mathrm{~nm}$ & 97 & 10 \\
\hline 16 & $\begin{array}{l}\text { Beeswax and } \\
\text { Carnauba } \\
\text { wax }\end{array}$ & Ketoprofen & Egg lecithin & Microemulsion ${ }^{16}$ & $150 \mathrm{~nm}$ & 97 & 26 \\
\hline 17 & Compritol & Ibuprofen & Pluronic $\AA$ F68 & $\begin{array}{l}\text { Hot-High pressure } \\
\text { homogenization } 17\end{array}$ & $<5 \mu \mathrm{m}$ & 98 & -- \\
\hline 18 & Beeswax & Ketoprofen & Tween ${ }^{\circledR} 80$ & Emulsion congealing ${ }^{18}$ & $790 \mu \mathrm{m}$ & 99 & -- \\
\hline 19 & Compritol & Ketoprofen & $\begin{array}{l}\text { Gelucire } \AA 50 / 13 \\
\text { Poloxamer } \AA 407 \text {, } \\
\text { Pluronic } \AA 127\end{array}$ & $\begin{array}{l}\text { Acoustic cavitation assisted hot } \\
\text { melt mixing (This work) }\end{array}$ & $\begin{array}{l}742 \mathrm{~nm} \\
676 \mathrm{~nm} \\
699 \mathrm{~nm}\end{array}$ & $\begin{array}{l}91 \\
87 \\
89 \\
\end{array}$ & $\begin{array}{l}12 \\
12 \\
12 \\
\end{array}$ \\
\hline
\end{tabular}

“--" indicates not reported. 
Table S2 Linear correlation coefficient $\left(\mathrm{R}^{2}\right)$ of fitting curves of in vitro drug release data of the formulated drug loaded solid lipid nanoparticles prepared using different stabilizers (GEL, POL, PLU) at different $\mathrm{pH}(4.0,7.4,10.0)$ in different release kinetic models.

\begin{tabular}{|l|l|l|l|l|l|l|}
\hline \multirow{2}{*}{ Formulation } & \multirow{2}{*}{$\mathbf{p H}$} & \multicolumn{5}{c|}{ Drug release kinetic models } \\
\cline { 2 - 7 } & & Zero & First & Higuchi & Korsmeyer-Peppas & Hixson-Crowell \\
\hline KP@CPT-GEL & 4.0 & 0.98073 & 0.98843 & 0.95314 & 0.99457 & 0.98628 \\
\hline KP@CPT-GEL & 7.4 & 0.98334 & 0.98778 & 0.93678 & 0.98168 & 0.98828 \\
\hline KP@CPT-GEL & 10.0 & 0.98155 & 0.93248 & 0.87669 & 0.9533 & 0.95501 \\
\hline KP@CPT-POL & 4.0 & 0.9628 & 0.96722 & 0.93494 & 0.93306 & 0.96608 \\
\hline KP@CPT-POL & 7.4 & 0.89119 & 0.92174 & 0.97058 & 0.92409 & 0.91311 \\
\hline KP@CPT-POL & 10.0 & 0.94844 & 0.94966 & 0.92595 & 0.9007 & 0.9528 \\
\hline KP@CPT-PLU & 4.0 & 0.98914 & 0.98613 & 0.89097 & 0.9776 & 0.98757 \\
\hline KP@CPT-PLU & 7.4 & 0.93024 & 0.95392 & 0.96365 & 0.94887 & 0.94857 \\
\hline KP@CPT-PLU & 10.0 & 0.97193 & 0.95253 & 0.90003 & 0.92223 & 0.96308 \\
\hline
\end{tabular}

\section{References}

(1) Thakkar, A.; Chenreddy, S.; Wang, J.; Prabhu, S. Evaluation of Ibuprofen Loaded Solid Lipid Nanoparticles and Its Combination Regimens for Pancreatic Cancer Chemoprevention. Int. J. Oncol. 2015, 46 (4), 1827-1834.

(2) Abu Amara, H. M. Preparation, Characterization and In Vitro Evaluation of Indomethacin Loaded Solid Lipid Nanoparticles. Jordan J. Pharm. Sci. 2014, 7 (1), 15-36.

(3) Kuo, Y.-C.; Chung, C.-Y. Solid Lipid Nanoparticles Comprising Internal Compritol 888 ATO, Tripalmitin and Cacao Butter for Encapsulating and Releasing Stavudine, Delavirdine and Saquinavir. Colloids Surfaces B. 2011, 88 (2), $682-690$.

(4) Dr. Abdul Hasan Sathali.A., Nisha. N. Development of Solid Lipid Nanoparticles of Rosuvastatin Calcium. BioMedRx. 2013, 1 (5), 536-548. 
(5) Malviya, N.; Somisetty, K.; Vemula, K.; Principal, V. Design and Development of a Novel Transmucosal Patch Embedded with Diclofenac Diethylamine Loaded Solid Lipid Nanoparticles. J. Young Pharm. 2015, 7 (1), 45-55.

(6) Palei, N. N.; Das, M. K. Preparation and characterization of Lornoxicam loaded solid lipid nanoparticles made from different lipids. Int. J. Pharm. Pharm. Sci. 2013, 5 (4), 438-442.

(7) Martínez, M. F.; Dáder, F. M.; López, R.; Reddy, S. P.; Moya, R. C.; Prabu, L. S.; Kumar, S. P. Fabrication and Characterization of Solid Lipid Microparticles of Ketoprofen. Ars Pharm 2011, 52 (1), 12-15.

(8) Kumar, R.; Singh, A.; Garg, N.; Siril, P. F. Solid Lipid Nanoparticles for the Controlled Delivery of Poorly Water Soluble NonSteroidal Anti-Inflammatory Drugs. Ultrason. Sonochem. 2018, 40, 686-696.

(9) Karami, M. A.; Sharif Makhmal Zadeh, B.; Koochak, M.; Moghimipur, E. Superoxide Dismutase-Loaded Solid Lipid Nanoparticles Prepared by Cold Homogenization Method: Characterization and Permeation Study Through Burned Rat Skin. Jundishapur J. Nat. Pharm. Prod. 2016, 11 (4), e33968.

(10) Khalil, R. M.; El-Bary, A. A.; Kassem, M. A.; Ghorab, M. M.; Ahmed, M. B. Solid lipid nanoparticles for topical delivery of meloxicam: development and in vitro characterization. European Scientific Journal. 2013, June Spec. Ed. Num. 3, 779-798.

(11) Aboud, H. M.; El komy, M. H.; Ali, A. A.; El Menshawe, S. F.; Abd Elbary, A. Development, Optimization, and Evaluation of Carvedilol-Loaded Solid Lipid Nanoparticles for Intranasal Drug Delivery. AAPS PharmSciTech 2016, 17 (6), $1353-1365$.

(12) Spada, G.; Gavini, E.; Cossu, M.; Rassu, G.; Giunchedi, P. Solid Lipid Nanoparticles with and without Hydroxypropyl- $\beta-$ Cyclodextrin: A Comparative Study of Nanoparticles Designed for Colonic Drug Delivery. Nanotechnology 2012, 23 (9), 095101.

(13) Priyanka, K.; Abdul Hasan, S. A. Preparation and Evaluation of Montelukast Sodium Loaded Solid Lipid Nanoparticles. J. Young Pharm. 2012, 4 (3), 129-137.

(14) Gönüllü, Ü.; Üner, M.; Yener, G.; Karaman, E. F.; Aydoğmuş, Z. Formulation and Characterization of Solid Lipid Nanoparticles, Nanostructured Lipid Carriers and Nanoemulsion of Lornoxicam for Transdermal Delivery. Acta Pharm. 2015, $65(1), 1-13$.

(15) Kheradmandnia, S.; Vasheghani-Farahani, E.; Nosrati, M.; Atyabi, F. Preparation and Characterization of Ketoprofen-Loaded Solid Lipid Nanoparticles Made from Beeswax and Carnauba Wax. Nanomed. Nanotechnol. Biol. Med. 2010, 6 (6), $753-759$. 
(16) Kheradmandnia, S.; Vasheghani-Farahani, E.; Nosrati, M.; Atyabi, F. The Effect of Process Variables on the Properties of Ketoprofen Loaded Solid Lipid nanoparticles of Beeswax and Carnauba Wax. Iran. J. Chem. Chem. Eng. 2010, 29(4), $181-187$.

(17) Sastre, V. G. S. E. Controlled Release Ibuprofen Nanoparticles: Physico-Chemical Characterization and Drug Release. Int. J. Pharm. Pharm. Sci. 2014, 6 (8), 99-107.

(18) Üner, M.; Gönüllü, Ü.; Yener, G.; Altınkurt, T. A New Approach for Preparing a Controlled Release Ketoprofen Tablets by Using Beeswax. IL Farmaco 2005, 60 (1), 27-31. 
